\title{
Error estimates for the unilateral buckling critical load of a thin plate
}

\author{
Mekki Ayadi*,** \\ * Laboratoire de Génie Mécanique, Ecole Nationale d'Ingénieurs de Monastir \\ Rue Ibn El Jazzar, 5019 Monastir, Tunisie \\ ** Département d'Informatique et de Mathématiques Appliquées \\ Institut Supérieur des Sciences Appliquées et de Technologie de Sousse \\ Cité Taffala, Ibn Khaldoun, 4003 Sousse, Tunisie \\ Mekki.Ayadi@enis.rnu.tn
}

ABSTRACT. The paper deals with error estimates for the unilateral buckling critical load of a thin plate in presence of an obstacle. First, using the Kirchhoff-Love's plate model, an abstract error estimate is given up. Its drawback is that it contains a hard term to evaluate. Then, by using the Mindlin's plate model together with a finite elements scheme of degree one, an error estimate, depending on the mesh size $h$, is established. The last part of the paper is devoted to some numerical results in order to validate the error estimate formula.

RÉSUMÉ. On s'intéresse, dans ce papier, à l'estimation de l'erreur pour la charge critique de flambement unilatéral d'une plaque mince en présence d'un obstacle. On donne d'abord une estimation abstraite avec le modèle de plaque de Kirchhoff-Love faisant intervenir un terme difficile à évaluer en fonction de la taille $h$ du maillage. Ensuite, le choix du modèle de plaque de Mindlin et l'emploi d'un élément fini de degré un permettent de donner une estimation de l'erreur en fonction de h. La fin du papier est consacrée à des résultats numériques qui ont permis de valider l'estimation d'erreur obtenue.

KEYWORDS: unilateral buckling, buckling critical load, finite element method, error estimate. MOTS-CLÉS : flambement unilatéral, charge critique de flambement, méthode d'éléments finis, estimation d'erreur.

DOI:10.3166/REMN.16.583-600 @ Lavoisier, Paris 


\section{Introduction}

Consider a thin plate of thickness $2 \varepsilon$ occupying a two-dimensional open set $\omega$. Assume that it is supported on the whole of its edge $\gamma$, clamped on a part $\gamma_{0}$ of its edge whose Lebesgue measure is not zero, and simply supported on $\gamma / \gamma_{0}$. Furthermore, the plate is subjected to a one-parameter plane compressive load $\lambda . g$ on another part $\gamma_{1}$ of its edge (see Figure 1).

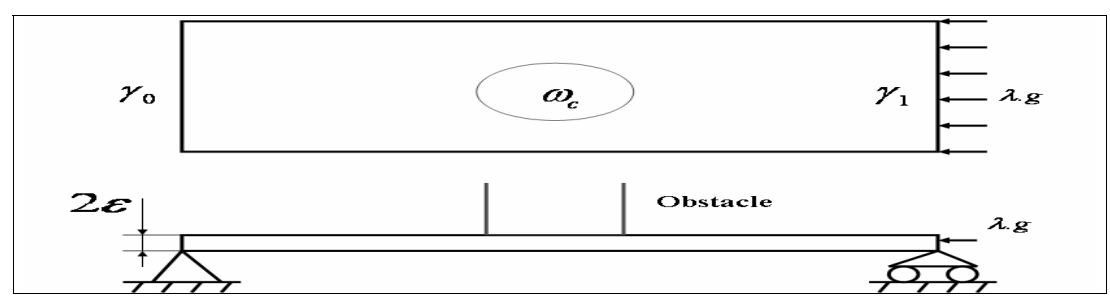

Figure 1. A rectangular plate is in presence of an obstacle

The plate is in presence of a rigid fixed plane obstacle that lies just above it (see Figure 1). The contact between the plate and the obstacle is supposed to be without friction. If the obstacle is not initially in contact with the plate, the problem becomes classical, in the sense that it has the same solution as the linear one. The unilateral buckling and even the unilateral post-buckling of thin plates have been tackled and investigated by many authors since the late seventies. Let us cite, in particular, (Do, 1975, 1976; Riddell, 1977; Cimetière, 1980, 1985; Kucera, 1982; Quittner, 1986; Goeleven et al., 1995; Shahwan et al., 1995; Goeleven et al., 1998; Smith et al., 1999, 2000; Chai, 2002). Recently, a small contribution to unilateral buckling problem was brought by Ayadi; he just suggested an algorithm in order to compute numerically the critical state of unilateral buckling problem (Ayadi, 2006).

The paper is organized as follows. The second section is devoted to the description of the unilateral buckling model. In the third section, the adequate framework so that the critical state of unilateral buckling exists, (see for instance (Do, 1975, 1976; Riddell, 1977)), is briefly recalled. In the fourth section, first, using the Kirchhoff-Love plate theory, an abstract error estimate is established. Its drawback is that it contains a term very hard to evaluate. Second, using the Mindlin plate theory together with a finite elements scheme of degree one, another error estimate, depending on the mesh size $h$, is achieved. In order to validate the numerical unilateral buckling model suggested: the Mindlin's plate model together with a finite element of degree one, a rectangular plate and a rectangular obstacle are considered in the fifth section. In the same figure are plotted three curves. The two first curves give the dependence of the buckling critical load, in the presence and in the absence of the obstacle respectively, upon the mesh size $h$. In order to check the 
error control, the third curve also gives the dependence (which is linear following the theoretical error estimate) of the difference of the two buckling critical loads (with and without contact) upon the mesh size $h$. The numerical results obtained are conformal with the theoretical ones.

\section{Mathematical modeling of unilateral buckling}

When taking into account the unilateral contact condition and considering a nonlinear elastic constitutive law, we obtain a very difficult mathematical problem (see (Ciarlet, 1986; Ciarlet et al., 1977; Duvaut et al., 1972). Nevertheless, we know a particular solution $u^{p}$ to the latter. It is the linear elasticity solution, obtained with linearized strains, for which the vertical displacement is zero (there is not bending) and the plane displacements are solution to the following variational equation:

$$
\sum_{\alpha, \beta, v, \mu=1}^{2} \int_{\omega} E_{\alpha \beta v \mu} \frac{\partial u_{v}^{p}}{\partial x_{\mu}} \frac{\partial v_{\alpha}}{\partial x_{\beta}} d \omega=\lambda \sum_{\alpha=1}^{2} \int_{\gamma_{1}} G_{\alpha} v_{\alpha} d \gamma \forall v \in V
$$

Where $E_{\alpha \beta \nu \mu}$ is the membrane stiffness tensor for the linear elastic constitutive law (depending on Young's modulus and the Poisson's ratio),

$$
G_{\alpha}=\int_{-\varepsilon}^{\varepsilon} g_{\alpha} d x_{3}, \alpha \in\{1,2\}
$$

$g_{1}$ and $g_{2}$ are the components of the plane compressive load $g$ (see Figure 1), and

$$
V=\left\{v \in H^{1}(\omega)^{2}: v=0 \text { on } \gamma_{0}\right\}
$$

The following usual assumptions are made.

(i) The boundary $\gamma$ of the bounded open set $\omega$ is supposed to be smooth enough.

(ii) $g_{1}, g_{2} \in L^{2}\left(\gamma_{1} \times\right]-\varepsilon, \varepsilon[)$ so that $G_{\alpha} \in L^{2}\left(\gamma_{1}\right), \alpha \in\{1,2\}$,

(iii) $E_{\alpha \beta v \mu} \in L^{\infty}(\omega)$ for all $(\alpha, \beta, \nu, \mu) \in\{1,2\}^{4}$,

(iv) $\exists k>0 ; \forall \theta \in \Theta_{s, 2} \sum_{\alpha, \beta, v, \mu=1}^{2} E_{\alpha \beta v \mu} \theta_{\alpha \beta} \theta_{v \mu} \geq k \sum_{\alpha, \beta=1}^{2}\left(\theta_{\alpha \beta}\right)^{2}$,

where $\Theta_{s, 2}$ denotes the set of all symmetric tensors of order two: that is the ellipticity property. It is shown in (Ciarlet, 1986) and in (Duvaut et al., 1972) that problem [1] 
admits a unique solution $u^{p} \in V$. Moreover, the tensor of membrane efforts is, (see for instance (Ayadi et al., 1990; Ayadi, 1993; Ciarlet et al., 1977), expressed by:

$$
n_{\alpha \beta}^{p}=\sum_{\nu, \mu=1}^{2} E_{\alpha \beta v \mu} \frac{\partial u_{v}^{p}}{\partial x_{\mu}}=\lambda . n_{\alpha \beta}^{g}, \quad \alpha, \beta \in\{1,2\} .
$$

Looking for a non-trivial solution to the nonlinear problem described above, we need the linearizing technique: set $u=u^{p}+w$ and show that the deflection $w_{3}$ of the plate is solution to the following inequality:

$$
\sum_{\alpha, \beta, v, \mu=1}^{2} \int_{\omega} D_{\alpha \beta v \mu} \frac{\partial^{2} w_{3}}{\partial x_{v} \partial x_{\mu}} \frac{\partial^{2}\left(v-w_{3}\right)}{\partial x_{\alpha} \partial x_{\beta}} d \omega \geq \lambda \sum_{\alpha, \beta=1}^{2} \int_{\omega} n_{\alpha \beta}^{g} \frac{\partial w_{3}}{\partial x_{\alpha}} \frac{\partial\left(v-w_{3}\right)}{\partial x_{\beta}} d \omega
$$

for all admissible deflection $v$, where $D_{\alpha \beta v \mu}$ denotes the bending rigidity tensor of the plate. The other components $w_{1}$ and $w_{2}$, of the displacement $w$, are related to the deflection $w_{3}$ by Kirchhoff-Love formulae (see Ciarlet et al., 1977).

\section{Mathematical framework and existence results}

Let us start by defining the adequate framework used in this paper so that the problem [2] admits at least one solution.

$$
\begin{gathered}
H=H_{0}^{1}(\omega), \\
W=\left\{v \in H^{2}(\omega): v=0 \text { on } \gamma \text { and } \frac{\partial v}{\partial n}=0 \text { on } \gamma_{0}\right\}, \\
K=\left\{v \in W: v \leq 0 \text { in } \omega_{c}\right\},
\end{gathered}
$$

$\omega_{c}$ being a subset of $\omega$ where the contact between the obstacle and the plate could occur, and $\partial v / \partial n$ is the derivative of the function $v$ with respect to the outward unit vector normal $n$ to the boundary $\gamma$ of $\omega$.

The Sobolev spaces $H$ and $W$ are respectively equipped with the following norms:

$$
|u|_{1, \omega}=\left(\int_{\omega} \sum_{\alpha=1}^{2}\left(\frac{\partial u}{\partial x_{\alpha}}\right)^{2}\right)^{\frac{1}{2}},\|u\|_{2, \omega}=\left(\int_{\omega} \sum_{|\alpha| \leq 2}\left(\partial^{|\alpha|} u\right)^{2}\right)^{\frac{1}{2}} .
$$

Let $a: W \times W \rightarrow I R$ and $b: H \times H \rightarrow I R$ be the bilinear forms defined by 


$$
\begin{gathered}
a(u, v)=\sum_{\alpha, \beta, v, \mu=1}^{2} \int_{\omega} D_{\alpha \beta v u} \frac{\partial^{2} u}{\partial x_{v} \partial x_{\mu}} \frac{\partial^{2} v}{\partial x_{\alpha} \partial x_{\beta}} d \omega, \\
b(u, v)=\sum_{\alpha, \beta=1}^{2} \int_{\omega} n_{\alpha \beta}^{g} \frac{\partial u}{\partial x_{\alpha}} \frac{\partial v}{\partial x_{\beta}} d \omega .
\end{gathered}
$$

They are obviously continuous in the spaces $H$ and $W$ respectively. So, there exist two positive constants $M$ and $N$ such that

$$
\begin{aligned}
& |a(u, v)| \leq M\|u\|_{2, \omega}\|v\|_{2, \omega}, \forall u, v \in W, \\
& |b(u, v)| \leq N\|u\|_{1, \omega}\|v\|_{1, \omega}, \forall u, v \in H .
\end{aligned}
$$

Assume now that the bending rigidity tensor $D_{\alpha \beta v \mu}$ satisfies the ellipticity property (iv) so that the bilinear form $a$ is $W$-elliptic (see (Ciarlet et al., 1977; Destuynder, 1990; Duvaut et al., 1972). That means there exists a positive constant $\alpha$ such that

$$
a(v, v) \geq \alpha\|v\|_{2, \omega}^{2}, \forall v \in W .
$$

Within the framework defined above, problem [2] is mathematically well posed as stated by the following theorem

Theorem 3.1. There exist $\lambda_{1}>0$ and nonzero vector $w_{3} \in K$ such that

$$
a\left(w_{3}, v-w_{3}\right) \geq \lambda_{1} b\left(w_{3}, v-w_{3}\right) \forall v \in K .
$$

Moreover, $\lambda_{1}$ is the minimum of the Rayleigh quotient over the closed convex cone $K$, which is realized on $w_{3}$ :

$$
\lambda_{1}=\min _{v \in K-\{0\}} \frac{a(v, v)}{b(v, v)}=a\left(w_{3}, w_{3}\right) .
$$

Proof: See (Riddell, 1977).

\section{Error estimates for the buckling critical load}

\subsection{The Kirchhoff-Love theory}

We shall approximate the "nonlinear eigenvalue problem" [2] by using a conformal finite element method. Let then $W_{h}$ be a finite-dimensional subspace of 
the space $W$ and let $K_{h}$ be a non empty closed convex subset of $W_{h}$, which is a cone. Observe that, in general, the set $K_{h}$ is not a subset of $K$.

The discrete "nonlinear eigenvalue problem", supposed to approach the continuous one [2], consists in finding pairs $\left(\lambda_{h}, w_{3 h}\right) \in I R_{+}^{*} \times\left(K_{h}-\{0\}\right)$ such that

$$
a\left(w_{3 h}, v_{h}-w_{3 h}\right) \geq \lambda_{h} b\left(w_{3 h}, v_{h}-w_{3 h}\right), \forall v_{h} \in K_{h}
$$

In fact, we are not interested in all pairs $\left(\lambda_{h}, w_{3 h}\right) \in I R_{+}^{*} \times\left(K_{h}-\{0\}\right)$ satisfying the variational inequality [5]. We just would like to focus in this paper on the smallest approximated buckling load $\lambda_{1 h}$ and the corresponding buckling mode $w_{3 h}$. Consequently, we handle the minimizing problem:

$$
\lambda_{1 h}=\min _{v_{h} \in K_{h}-\{0\}} \frac{a\left(v_{h}, v_{h}\right)}{b\left(v_{h}, v_{h}\right)} .
$$

Obviously, the minimizing problem [6] is mathematically well posed. That means: there exists a function $w_{3 h} \in K_{h}-\{0\}$, which realizes the minimum of problem [6].

From now on, we shall choose $b\left(w_{3}, w_{3}\right)=b\left(w_{3 h}, w_{3 h}\right)=1$, for all $h>0$. Furthermore, if the tensor of membrane efforts $n_{\alpha \beta}^{g}$ verifies the ellipticity property (as in our case), there exists a positive constant $\eta>0$ such that

$$
\sum_{\alpha, \beta=1}^{2} n_{\alpha \beta}^{g} \theta_{\alpha} \theta_{\beta} \geq \eta \sum_{\alpha=1}^{2} \theta_{\alpha}^{2}
$$

for all vector $\theta \in I R^{2}$, then the embedding of the space $W$ in the space $H$, supposed to be equipped with the norm $|\cdot|_{b}: H \rightarrow I R_{+}: v \rightarrow|v|_{b}=\sqrt{b(v, v)}$, is continuous (Raviart et al., 1983).

Lemma 4.1. Let $y_{h}=P_{K_{h}} w_{3}$ be the projection, in the sense of the inner product defined by the bilinear forma(.,.), of the exact buckling mode $w_{3}$ on the convex set $K_{h}$. And let $z_{h}=P_{K} w_{3 h}$ be the projection, also in the sense of $a(.,$.$) , of the$ approximated buckling mode $w_{3 h}$ on the convex set $K$. Then we have the two following inequalities: 


$$
\begin{gathered}
\left\|w_{3}-P_{K_{h}} w_{3}\right\|_{2, \omega} \leq \frac{M}{\alpha} \inf _{w_{h} \in K_{h}}\left\|w_{3}-w_{h}\right\|_{2, \omega}, \\
\left\|w_{3 h}-P_{K} w_{3 h}\right\|_{2, \omega} \leq \frac{M}{\alpha} \inf _{w \in K}\left\|w_{3 h}-w\right\|_{2, \omega} .
\end{gathered}
$$

Furthermore, there exists a positive real $h_{0}$ such that

$$
\begin{aligned}
& \left(\lim _{h \rightarrow 0} \inf _{w_{h} \in K_{h}}\left\|w_{3}-w_{h}\right\|_{2, \omega}=0\right) \Rightarrow\left(h \leq h_{0} \Rightarrow \frac{1}{\left|P_{K_{h}} w_{3}\right|_{b}} \leq 2\right), \\
& \left(\liminf _{h \rightarrow 0}\left\|w_{w \in K}-w\right\|_{2, \omega}=0\right) \Rightarrow\left(h \leq h_{0} \Rightarrow \frac{1}{\left|P_{K} w_{3 h}\right|_{b}} \leq 2\right) .
\end{aligned}
$$

Proof: According to the definitions made in the lemma 4.1, we have:

$$
a\left(w_{3}-P_{K_{h}} w_{3}, w_{h}-P_{K_{h}} w_{3}\right) \leq 0, \forall w_{h} \in K_{h} .
$$

Using the $W$-ellipticity and then the continuity of the bilinear form $a$ (with usual constants $\alpha$ and $M$ ), together with [11], we obtain, for all $w_{h} \in K_{h}$,

$$
\begin{aligned}
\alpha\left\|w_{3}-P_{K_{h}} w_{3}\right\|_{2, \omega}^{2} & \leq a\left(w_{3}-P_{K_{h}} w_{3}, w_{3}-P_{K_{h}} w_{3}\right) \\
& =a\left(w_{3}-P_{K_{h}} w_{3}, w_{3}-w_{h}\right)+a\left(w_{3}-P_{K_{h}} w_{3}, w_{h}-P_{K_{h}} w_{3}\right) \\
& \leq a\left(w_{3}-P_{K_{h}} w_{3}, w_{3}-w_{h}\right) \\
& \leq M\left\|w_{3}-P_{K_{h}} w_{3}\right\|_{2, \omega}\left\|w_{3}-w_{h}\right\|_{2, \omega} .
\end{aligned}
$$

Hence the inequality [7] is showed.

Suppose that $\lim _{h \rightarrow 0} \inf _{w_{h} \in K_{h}}\left\|w_{3}-w_{h}\right\|_{2, \omega}=0$. Then, according to [7], the sequence $\left(P_{K_{h}} w_{3}\right)$ converges to $w_{3}$ in the space $W$. Since the embedding of the space $W$ in the space $H$ is continuous, the sequence $\left(\left|P_{K_{h}} w_{3}\right|_{b}\right)$ converges to $\left|w_{3}\right|_{b}=1$. Hence the inequality [9] is showed. The results [8] and [10] are exactly showed in the same way. 


\section{Lemma 4.2. Suppose that}

$$
\lim _{h \rightarrow 0} \inf _{w_{h} \in K_{h}}\left\|w_{3}-w_{h}\right\|_{2, \omega}=0
$$

Then the sequence $\left(w_{3 h}\right)_{h>0}$ is bounded in the norm $\|\cdot\|_{2, \omega}$.

Proof: Using first the $W$-ellipticity of $a$, and then the fact that the projection on a convex set is a Lipschitz mapping with unit ratio, we obtain:

$$
\begin{aligned}
\alpha\left\|w_{3 h}\right\|_{2, \omega}^{2} & \leq a\left(w_{3 h}, w_{3 h}\right) \\
& =\inf _{v_{h} \in K_{h}-\{0\}} \frac{a\left(v_{h}, v_{h}\right)}{b\left(v_{h}, v_{h}\right)} \\
& \leq \frac{1}{\left|P_{K_{h}} w_{3}\right|_{b}^{2}} a\left(P_{K_{h}} w_{3}, P_{K_{h}} w_{3}\right) \\
& \leq \frac{1}{\left|P_{K_{h}} w_{3}\right|_{b}^{2}} a\left(w_{3}, w_{3}\right) .
\end{aligned}
$$

Finally, propriety [9] and the continuity of the bilinear form $a$ yield

$$
\left\|w_{3 h}\right\|_{2, \omega} \leq 2 \sqrt{\frac{M}{\alpha}}\left\|w_{3}\right\|_{2, \omega} .
$$

We are now in a position to prove an abstract error estimate for the unilateral buckling critical load of a thin plate, with Kirchhoff-Love theory, in presence of an obstacle.

Theorem 4.3. Suppose that

$$
\lim _{h \rightarrow 0} \inf _{w_{h} \in K_{h}}\left\|w_{3}-w_{h}\right\|_{2, \omega}=\lim _{h \rightarrow 0} \inf _{w \in K}\left\|w_{3 h}-w\right\|_{2, \omega}=0 .
$$

Then there exist four positive constants, $c_{1}^{\prime}, c_{2}^{\prime}, c_{1}$ and $c_{2}$ independent of the subspace $W_{h}$ and of the convex cone $K_{h}$ such that

$$
-c_{1}^{\prime} \inf _{w \in K}\left\|w_{3 h}-w\right\|_{2, \omega}^{2}-c_{2}^{\prime} \inf _{w \in K}\left\|w_{3 h}-w\right\|_{2, \omega} \leq \lambda_{1 h}-\lambda_{1} \leq c_{1} \inf _{w_{h} \in K_{h}}\left\|w_{3}-w_{h}\right\|_{2, \omega}^{2}+c_{2} \inf _{w_{h} \in K_{h}}\left\|w_{3}-w_{h}\right\|_{2, \omega}
$$


Proof: First, we show the right hand side of the double inequality [13]. Let $v_{h} \in K_{h}-\{0\}$, following the expressions [4] of the exact buckling critical load, and [6] of the approximated one, we have:

$$
\begin{aligned}
\lambda_{1 h}-\lambda_{1} & \leq \frac{a\left(v_{h}, v_{h}\right)}{b\left(v_{h}, v_{h}\right)}-a\left(w_{3}, w_{3}\right) \\
& =a\left(\frac{v_{h}}{\left|v_{h}\right|_{b}}-w_{3}, \frac{v_{h}}{\left|v_{h}\right|_{b}}-w_{3}\right)+2 a\left(w_{3}, \frac{v_{h}}{\left|v_{h}\right|_{b}}-w_{3}\right)
\end{aligned}
$$

Hence

$$
\lambda_{1 h}-\lambda_{1} \leq M\left\|\frac{v_{h}}{\left|v_{h}\right|_{b}}-w_{3}\right\|_{2, \omega}^{2}+2 M\left\|w_{3}\right\|_{2, \omega}\left\|\frac{v_{h}}{\left|v_{h}\right|_{b}}-w_{3}\right\|_{2, \omega} .
$$

But the inequality [14] is in particular hold for $v_{h}=P_{K_{h}} w_{3}$. Then, the continuity of the embedding of $W$ in $H$ (with constant $c$ ) yields:

$$
\begin{aligned}
\lambda_{1 h}-\lambda_{1} \leq M & \left\|\frac{P_{K_{h}} w_{3}}{\left|P_{K_{h}} w_{3}\right|_{b}}-w_{3}\right\|_{2, \omega}^{2}+2 M\left\|w_{3}\right\|_{2, \omega}\left\|\frac{P_{K_{h}} w_{3}}{\left|P_{K_{h}} w_{3}\right|_{b}}-w_{3}\right\|_{2, \omega} \\
= & \frac{M}{\left|P_{K_{h}} w_{3}\right|_{b}^{2}}\left\|P_{K_{h}} w_{3}-\left|P_{K_{h}} w_{3}\right|_{b} w_{3}\right\|_{2, \omega}^{2}+\frac{2 M}{\left|P_{K_{h}} w_{3}\right|_{b}}\left\|w_{3}\right\|_{2, \omega}\left\|P_{K_{h}} w_{3}-\left|P_{K_{h}} w_{3}\right|_{b} w_{3}\right\|_{2, \omega} \\
= & \frac{M}{\left|P_{K_{h}} w_{3}\right|_{b}^{2}}\left\|\left(P_{K_{h}} w_{3}-w_{3}\right)+\left(\left|w_{3}\right|_{b}-\left|P_{K_{h}} w_{3}\right|_{b} w_{3}\right)\right\|_{2, \omega}^{2}+ \\
& \frac{2 M}{\left|P_{K_{h}} w_{3}\right|_{b}}\left\|w_{3}\right\|_{2, \omega}\left\|\left(P_{K_{h}} w_{3}-w_{3}\right)+\left(\left|w_{3}\right|_{b}-\left|P_{K_{h}} w_{3}\right|_{b} w_{3}\right)\right\|_{2, \omega} \\
\leq & \frac{M}{\left|P_{K_{h}} w_{3}\right|_{b}^{2}}\left(1+c\left\|w_{3}\right\|_{2, \omega}\right)^{2}\left\|P_{K_{h}} w_{3}-w_{3}\right\|_{2, \omega}^{2}+ \\
& \frac{2 M}{\left|P_{K_{h}} w_{3}\right|_{b}}\left\|w_{3}\right\|_{2, \omega}\left(1+c\left\|w_{3}\right\|_{2, \omega}\right) \| P_{K_{h}} w_{3}-\left.w_{3}\right|_{2, \omega} .
\end{aligned}
$$

According to lemma 4.1 or [7] and [9], we obtain the right inequality of [13]: for $h \leq h_{0}$, 


$$
\lambda_{1 h}-\lambda_{1} \leq \frac{4 M^{3}}{\alpha^{2}}\left(1+c\left\|w_{3}\right\|_{2, \omega}\right)^{2} \inf _{w_{h} \in K_{h}}\left\|w_{3}-w_{h}\right\|_{2, \omega}^{2}+\frac{4 M^{2}}{\alpha}\left\|w_{3}\right\|_{2, \omega}\left(1+c\left\|w_{3}\right\|_{2, \omega}\right) \inf _{w_{h} \in K_{h}}\left\|w_{3}-w_{h}\right\|_{2, \omega}
$$

Second, we show the left hand side of the double inequality [13]. Let $v \in K-\{0\}$, following again the expressions [4] of the exact buckling critical load, and [6] of the approximated one, we have:

$$
\begin{aligned}
\lambda_{1 h}-\lambda_{1} & \geq a\left(w_{3 h}, w_{3 h}\right)-\frac{a(v, v)}{b(v, v)} \\
& =a\left(w_{3 h}-\frac{v}{|v|_{b}}, \frac{v}{|v|_{b}}-w_{3 h}\right)+2 a\left(w_{3 h}, w_{3 h}-\frac{v}{|v|_{b}}\right) .
\end{aligned}
$$

Hence

$$
\lambda_{1 h}-\lambda_{1} \geq-M\left\|w_{3 h}-\frac{v}{|v|_{b}}\right\|_{2, \omega}^{2}-2 M\left\|w_{3 h}\right\|_{2, \omega}\left\|w_{3 h}-\frac{v}{|v|_{b}}\right\|_{2, \omega} .
$$

The inequality [15] is hold, in particular, for $v=P_{K} w_{3 h}$. Consequently, we have:

$$
\begin{aligned}
\lambda_{1 h}-\lambda_{1} \geq & \frac{-M}{\left|P_{K} w_{3 h}\right|_{b}^{2}}\left\|P_{K} w_{3 h}-\left|P_{K} w_{3 h}\right|_{b} w_{3 h}\right\|_{2, \omega}^{2}+\frac{-2 M}{\left|P_{K} w_{3 h}\right|_{b}}\left\|w_{3 h}\right\|_{2, \omega}\left\|P_{K} w_{3 h}-\left|P_{K} w_{3 h}\right|_{b} w_{3 h}\right\|_{2, \omega} \\
= & \frac{-M}{\left|P_{K} w_{3 h}\right|_{b}^{2}}\left\|\left(P_{K} w_{3 h}-w_{3 h}\right)+\left(\left|w_{3 h}\right|_{b}-\left|P_{K} w_{3 h}\right|_{b}\right) w_{3 h}\right\|_{2, \omega}^{2}+ \\
& \frac{-2 M}{\left|P_{K} w_{3 h}\right|_{b}}\left\|w_{3 h}\right\|_{2, \omega}\left\|\left(P_{K} w_{3 h}-w_{3 h}\right)+\left(\left|w_{3 h}\right|_{b}-\left|P_{K} w_{3 h}\right|_{b}\right) w_{3 h}\right\|_{2, \omega} \\
\geq & \frac{-M}{\left|P_{K} w_{3 h}\right|_{b}^{2}}\left(1+c\left\|w_{3 h}\right\|_{2, \omega}\right)^{2}\left\|P_{K} w_{3 h}-w_{3 h}\right\|_{2, \omega}^{2}+ \\
& \frac{-2 M}{\left|P_{K} w_{3 h}\right|_{b}}\left\|w_{3 h}\right\|_{2, \omega}\left(1+c\left\|w_{3 h}\right\|_{2, \omega}\right)\left\|P_{K} w_{3 h}-w_{3 h}\right\|_{2, \omega} .
\end{aligned}
$$

Hence, following to [8], [10] and [12], we obtain the left inequality of [13]: for $h \leq h_{0}$,

$$
\lambda_{1 h}-\lambda_{1} \geq \frac{-4 M^{3}}{\alpha^{2}}\left(1+2 c \sqrt{\frac{M}{\alpha}}\left\|w_{3}\right\|_{2, \omega}\right)^{2} \inf _{n \in K}\left\|w_{3 h}-w\right\|_{2, \omega}^{2}-\frac{8 M^{\frac{5}{2}}}{\alpha^{\frac{3}{2}}}\left\|w_{3}\right\|_{2, \omega}\left(1+2 c \sqrt{\frac{M}{\alpha}}\left\|w_{3}\right\|_{2, \omega}\right) \underset{\omega n \in K}{\inf }\left\|w_{3 h}-w\right\|_{2, \omega}
$$


REMARK. - Several comments are in order about this theorem.

(i) We would like to remind here that, in the linear case $K=W$ (buckling without obstacle), the abstract error estimate for the buckling critical load is much more simpler as exemplifies its expression (Ciarlet, 1978):

$$
\left|\lambda_{1 h}-\lambda_{1}\right| \leq C_{1} \cdot \inf _{w_{h} \in K_{h}}\left\|w_{3}-w_{h}\right\|_{2, \omega}^{2} .
$$

(ii) Observe that the approximation method in the linear case is more accurate than that of nonlinear case.

(iii) If the inclusion $K_{h} \subset K$ holds, then obviously the term $\inf _{w \in K}\left\|w_{3 h}-w\right\|_{2, \omega}$, which is expected to be the hardest to evaluate (Ciarlet, 1978), vanishes in the error estimate formula [13],

$$
\left|\lambda_{1 h}-\lambda_{1}\right| \leq C_{1} . \inf _{w_{h} \in K_{h}}\left\|w_{3}-w_{h}\right\|_{2, \omega}^{2}+C_{2} . \inf _{w_{h} \in K_{h}}\left\|w_{3}-w_{h}\right\|_{2, \omega}
$$

\subsection{The Mindlin theory}

In order to minimize the number of degrees of freedom, the Kirchhoff-Love's plate model is often replaced by the Mindlin's one. The later satisfies the exact three-dimensional boundary conditions, but does not allow representing threedimensional singularities. Boundary layer models based on Kirchhoff-Love theory, at the opposite, permit to point out the existence of such stress singularities (Davet et al., 1985). Fortunately, the two theories are suitable for computing the critical state of buckling. The Mindlin's plate model involves, as unknowns, the deflection $w_{3}$ and the two rotations $\theta_{1}$ and $\theta_{2}$ of the mid plane of the plate, which are related by the formulae (Ciarlet et al., 1977), (Destuynder, 1990):

$$
\theta_{\alpha}=-\frac{\partial w_{3}}{\partial x_{\alpha}}, \alpha=1,2
$$

The changing of the plate model obviously involves a changing of the framework:

$$
\begin{gathered}
H_{M}=\left\{v=\left(v_{3}, r_{1}, r_{2}\right) \in H^{1}(\omega) \times\left(L^{2}(\omega)\right)^{2}: v_{3}=0 \text { on } \gamma_{0}\right\}, \\
W_{M}=\left\{v=\left(v_{3}, r_{1}, r_{2}\right) \in\left(H^{1}(\omega)\right)^{3}: v_{3}=r_{1}=r_{2}=0 \text { on } \gamma_{0}\right\}, \\
K_{M}=\left\{v \in W_{M}: v_{3} \leq 0 \text { in } \omega_{c}\right\},
\end{gathered}
$$


$\omega_{c}$ being the contact region. The sobolev spaces $W_{M}$ and $H_{M}$ are respectively equipped with the following norms:

$$
\|v\|_{W_{M}}=\left(\left\|v_{3}\right\|_{1, \omega}^{2}+\left\|r_{1}\right\|_{1, \omega}^{2}+\left\|r_{2}\right\|_{1, \omega}^{2}\right)^{\frac{1}{2}},\|v\|_{H_{M}}=\left(\left\|v_{3}\right\|_{1, \omega}^{2}+\left\|r_{1}\right\|_{0, \omega}^{2}+\left\|r_{2}\right\|_{0, \omega}^{2}\right)^{\frac{1}{2}} .
$$

Let now $a_{M}$ and $b_{M}$ be the following bilinear forms defined in the spaces $W_{M}$ and $H_{M}$ respectively by:

$$
\begin{gathered}
a_{M}(u, v)=\sum_{\alpha, \beta, v, \mu=1}^{2} \int_{\omega} D_{\alpha \beta \gamma u} \frac{\partial \theta_{\alpha}}{\partial x_{\beta}} \frac{\partial r_{v}}{\partial x_{\mu}} d \omega+\sum_{\alpha, \beta=1}^{2} \int_{\omega} G_{\alpha \beta}\left(\theta_{\alpha}+\frac{\partial w_{3}}{\partial x_{\alpha}}\right)\left(r_{\beta}+\frac{\partial v_{3}}{\partial x_{\beta}}\right) d \omega, \\
b_{M}(u, v)=\frac{1}{2} \sum_{\alpha, \beta=1}^{2} \int_{\omega} n_{\alpha \beta}^{g} \frac{\partial w_{3}}{\partial x_{\alpha}} \frac{\partial v_{3}}{\partial x_{\beta}} d \omega+\frac{1}{2} \sum_{\alpha, \beta=1}^{2} \int_{\omega} n_{\alpha \beta}^{8} \theta_{\alpha} r_{\beta} d \omega,
\end{gathered}
$$

Where $G_{\alpha \beta}$ denotes the transverse shear rigidity tensor, which verify the same ellipticity property as the tensor $n_{\alpha \beta}^{g}$. The bilinear forms $a_{M}$ and $b_{M}$ are obviously continuous in the spaces $W_{M}$ and $H_{M}$ respectively. Then, the mapping $|\cdot|_{b_{M}}: H \rightarrow I R_{+}: v \rightarrow|v|_{b_{M}}=\sqrt{b_{M}(v, v)}$ is a norm equivalent to the norm $\|\cdot\|_{H_{M}}$, and consequently the embedding of the space $W_{M}$ in the space $H_{M}$, supposed to be equipped with the norm $|\cdot|_{b_{M}}$, is continuous. Finally, the bilinear form $a_{M}$ is $W_{M}$-elliptic, see (Ciarlet et al., 1977; Destuynder, 1990; Duvaut et al., 1972).

Within the framework defined above, the unilateral buckling problem for the Mindlin theory,

$$
a_{M}(u, v-u) \geq \lambda_{M 1} b_{M}(u, v-u), \forall v \in K_{M},
$$

which is equivalent to the minimizing problem

$$
\lambda_{M 1}=\min _{v \in K_{M}-\{0\}} \frac{a_{M}(v, v)}{b_{M}(v, v)}
$$

is, by virtue of theorem 2.1 , mathematically well posed. Let $u=\left(w_{3}, \theta_{1}, \theta_{2}\right)$ be its solution.

Because the Mindlin's model only involves first order partial derivatives, a continuous finite element is used. This is the triangle with three nodes. Let $\left(\mathscr{J}_{h}\right)$ be a 
regular triangulation. Then the space $W_{M}$ and the convex set $K_{M}$ are respectively approximated by

$W_{M h}=\left\{\left(v_{3 h}, r_{1 h}, r_{2 h}\right) \in\left(C^{0}(\bar{\omega})\right)^{3}: v_{3 h \mid}, r_{1 h \mid T}, r_{2 h \mid T} \in \mathscr{P}_{1}[X, Y], T \in\left(\mathscr{J}_{h}\right)\right.$ and $v_{3 h}=r_{1 h}=r_{2 h}=0$ on $\left.\gamma_{0}\right\}$,

And by

$$
K_{M h}=\left\{\left(v_{3 h}, r_{1 h}, r_{2 h}\right) \in W_{M h}: v_{3 h}\left(x_{i}, y_{j}\right) \leq 0, \text { for all mesh node }\left(x_{i}, y_{j}\right) \in \bar{\omega}_{c}\right\},
$$

where

$$
\mathscr{P}_{1}[X, Y]=\left\{P_{1}=a_{00}+a_{10} X+a_{01} Y: a_{00}, a_{10}, a_{01} \in I R\right\}
$$

is the space of polynomials of degree less or equal one.

It is obvious that the approximated unilateral buckling problem,

$$
\lambda_{M 1 h}=\min _{v_{h} \in K_{M h}-\{0\}} \frac{a_{M}\left(v_{h}, v_{h}\right)}{b_{M}\left(v_{h}, v_{h}\right)},
$$

is well posed (the minimum of a continuous cost over a compact set). Let $u_{h}=\left(w_{3 h}, \theta_{1 h}, \theta_{2 h}\right)$ be its solution. Moreover, we have the following error estimate.

Corollary 4.4. Suppose that $u=\left(w_{3}, \theta_{1}, \theta_{2}\right) \in\left(H^{2}(\omega)\right)^{3}$. Then, there exist two positive constants $c_{1}$ and $c_{2}$ independent of the subspace $W_{M h}$ and of the convex cone $K_{M h}$ such that

$$
\left|\lambda_{M 1 h}-\lambda_{M 1}\right| \leq c_{1} h^{2}+c_{2} h
$$

$$
\text { If } K_{M h}=W_{M h} \text {, the error control [20] reduces to }
$$

$$
\left|\lambda_{M 1 h}-\lambda_{M 1}\right| \leq c_{2} h^{2}
$$

Proof: First of all, we have $K_{M h} \subset K_{M}$, which involves $\lambda_{M 1 h}-\lambda_{M 1} \geq 0$. Second, we shall choose $b_{M}(u, u)=b_{M}\left(u_{h}, u_{h}\right)=1$, for all $h>0$. Third, we have the same conditions as theorem 4.3: the bilinear forms $a_{M}$ and $b_{M}$ are continuous, the bilinear form $a_{M}$ is $W_{M}$-elliptic and the embedding of the space $W_{M}$ in the 
space $H_{M}$, supposed to be equipped with the norm $|\cdot|_{b_{M}}$, is continuous. Consequently, if $\lim _{h \rightarrow 0} \inf _{v_{h} \in K_{M h}}\left\|u-v_{h}\right\|_{W_{M}}=0$ then there exist two positive constants $c_{1}^{\prime}$ and $c_{2}^{\prime}$ independent of the subspace $W_{M h}$ and of the convex cone $K_{M h}$ such that

$$
\left|\lambda_{M 1 h}-\lambda_{M 1}\right| \leq c_{1}^{\prime} \cdot \inf _{v_{h} \in K_{M h}}\left\|u-v_{h}\right\|_{W_{M}}^{2}+c_{2}^{\prime} \cdot \inf _{v_{h} \in K_{M h}}\left\|u-v_{h}\right\|_{W_{M}}
$$

Finally, with a regular triangulation $\left(\mathscr{J}_{h}\right)$ (see for instance (Raviart et al., 1983)), there exists a positive constant $c_{3}^{\prime}$ such that

$$
\inf _{v_{h} \in K_{M h}}\left\|u-v_{h}\right\|_{W_{M}} \leq c_{3}^{\prime} h|u|_{2, \omega},
$$

which achieves the error estimate [20].

$$
\begin{aligned}
& \text { If } K_{M h}=W_{M h}, \text { for all } v_{h} \in K_{M h}-\{0\}, \text { we have } \\
& \begin{aligned}
0 \leq \lambda_{M 1 h}-\lambda_{M 1} \leq & \frac{a_{M}\left(v_{h}, v_{h}\right)}{b_{M}\left(v_{h}, v_{h}\right)}-a_{M}(u, u) \\
& =a_{M}\left(\frac{v_{h}}{\left|v_{h}\right|_{b_{M}}}-u, \frac{v_{h}}{\left|v_{h}\right|_{b_{M}}}-u\right)+2 a_{M}\left(u, \frac{v_{h}}{\left|v_{h}\right|_{b_{M}}}-u\right)
\end{aligned}
\end{aligned}
$$

The error estimate [21] is due to the fact that

$$
a_{M}\left(u, \frac{v_{h}}{\left|v_{h}\right|_{b_{M}}}-u\right) \leq 0
$$

\section{Numerical results}

In order to validate our numerical unilateral buckling model, the following numerical test is handled.

The plate, occupying the two-dimensional domain $\omega=]-0.1,0.1[\times]-0.05,0.05[$, is simply supported on the whole of its edge and compressed on the part $\gamma_{1}$ of its edge by a uniform load $2 \varepsilon \lambda$. Furthermore, the plate is supposed to have a thickness $2 \varepsilon=0.006 \mathrm{~m}$ and to be made of an elastic, homogenous and isotropic 
material whose mechanical features are: the Young's modulus $E=1.000 \mathrm{e}+09 P a$, and the Poisson's ratio $v=0.3$ (see Figure 2).

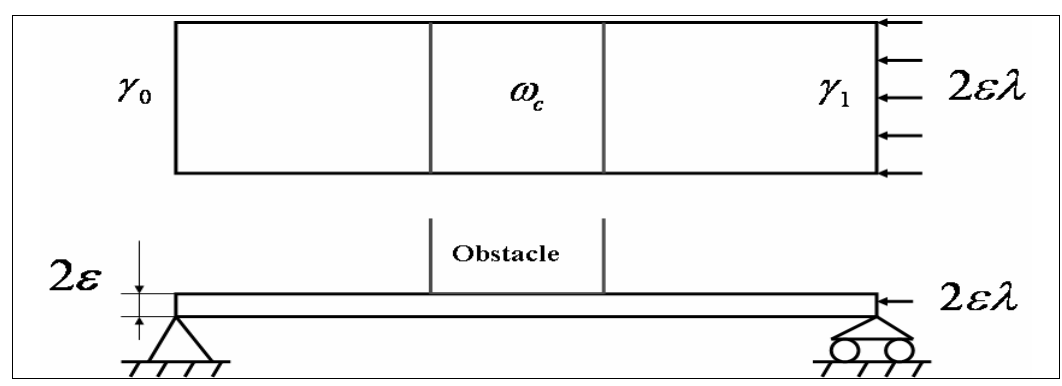

Figure 2. The rectangular plate is in presence of the obstacle

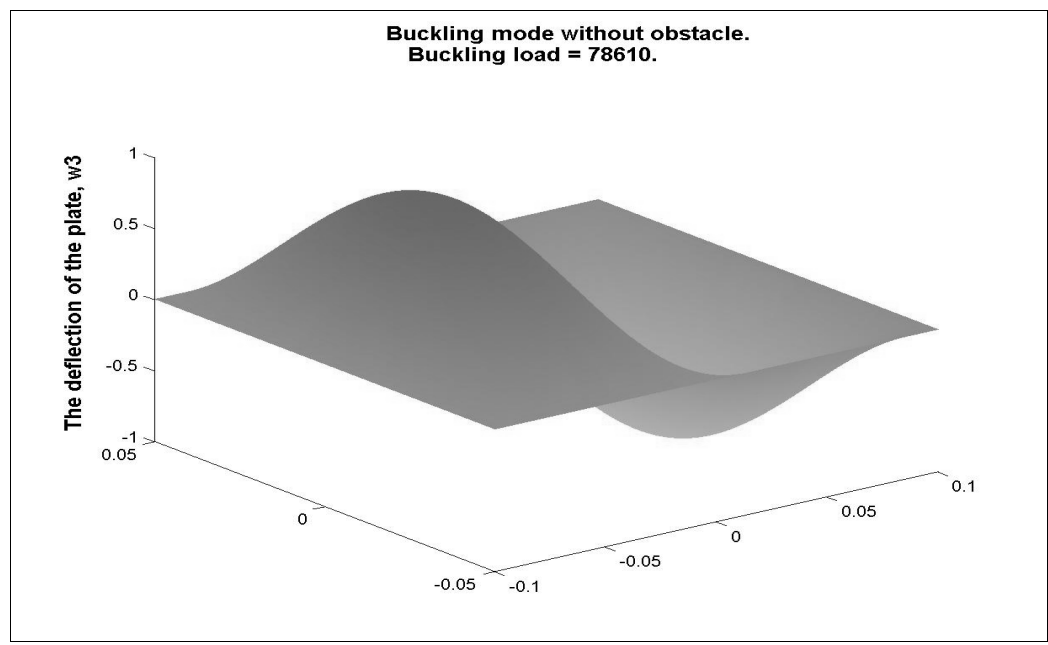

Figure 3. The buckling mode of the plate in the absence of any obstacle

Consider now the obstacle occupying the rectangular domain:

$\bar{\omega}_{c}=[-0.04,0.04] \times[-0.02,0.02]$. 


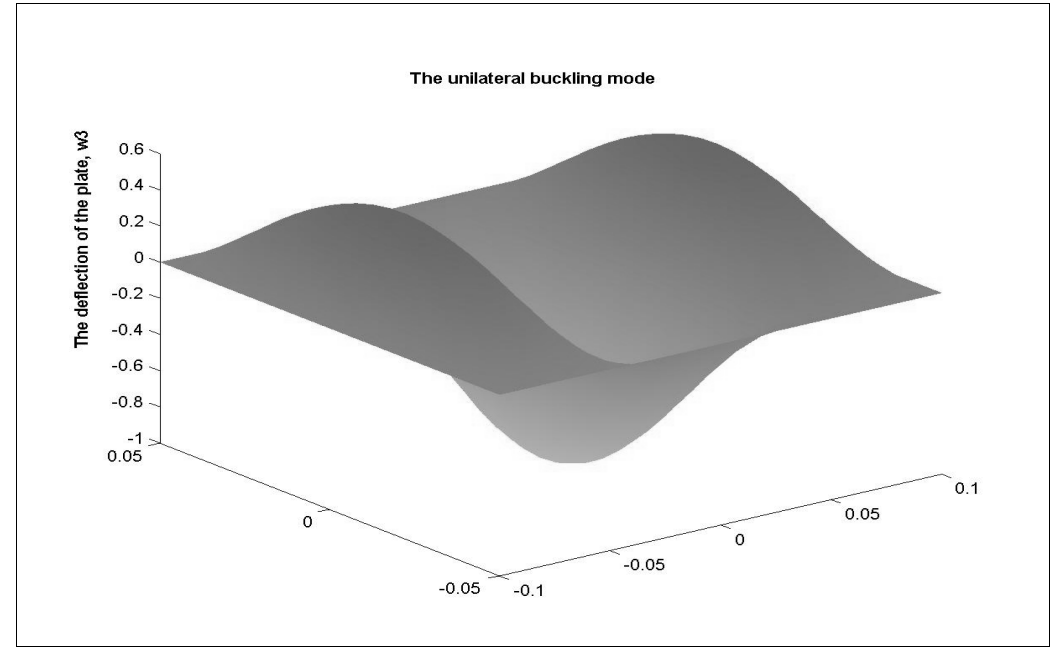

Figure 4. The unilateral buckling mode of the plate

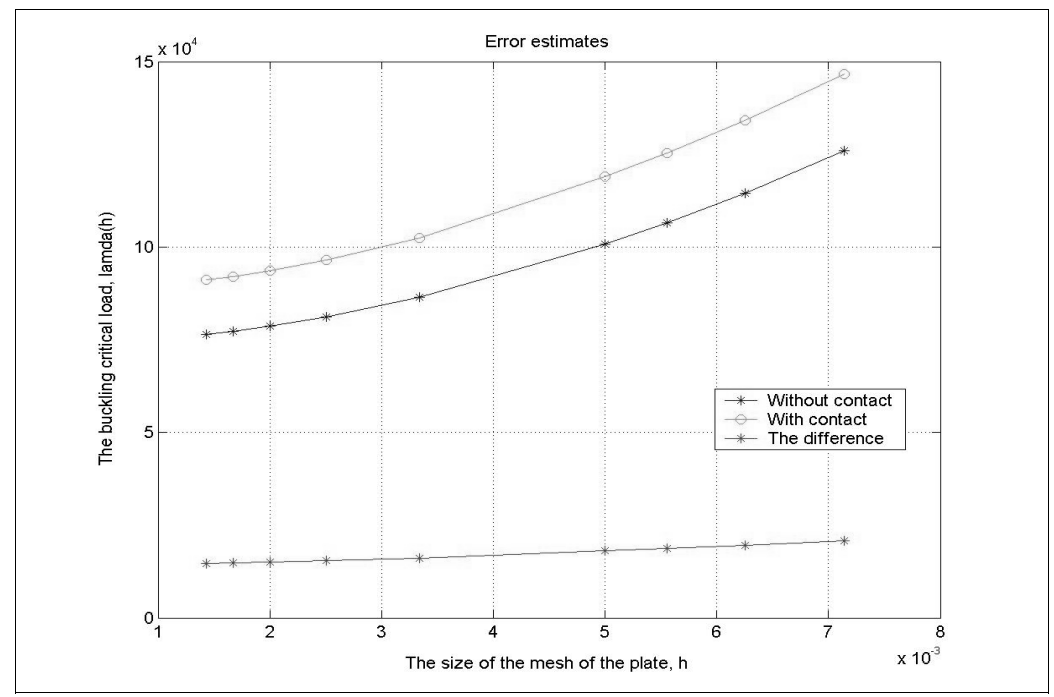

Figure 5. The dependence of the critical load upon the mesh size

The two first curves above give the dependence of the buckling critical load, in the presence and in the absence of the obstacle respectively, upon the mesh size. Following theoretical error estimate formulas [20] and [21], the difference between the two critical loads (with and without contact) must be linear as a function of the mesh size. The third curve shows that this linear behavior is numerically confirmed. 


\section{Conclusion and perspectives}

Using the Mindlin plate theory together with a finite element method of very low degree (linear local interpolation) is sufficient to approach the unilateral buckling critical load of a thin plate in presence of an obstacle, as exemplify both of the theoretical error estimate and the obtained numerical results.

The Kirchhoff-Love plate theory is not only difficult to implement numerically, but also leads to the abstract error estimate [13] whose left hand side is very hard to evaluate. Using the very interesting result, namely the maximum of two functions in $H^{1}(\omega)$ is also in $H^{1}(\omega)$ (Lewy et al., 1969); Ciarlet has succeeded, in the equilibrium position of an elastic membrane problem in presence of an obstacle, to evaluate the term $\inf _{v \in K}\left\|u_{h}-v\right\|_{1, \omega}$ (Ciarlet, 1978). So, if the maximum of two functions in $H^{2}(\omega)$ is also in $H^{2}(\omega)$ (which is not true for instance if $\omega$ is an open subset of $I R$ ), then there is a hope to evaluate the term $\inf _{w \in K}\left\|w_{3 h}-w\right\|_{2, \omega}$.

In the future work, we are going to deal with achieving an error estimate for the unilateral buckling mode by using the Mindlin plate theory.

It is a pleasure to thank Professor Hedi BelHadj Salah, from the "'Laboratory of Mechanical Engineering', at National Engineering School of Monastir, for so many fruitful discussions and for his precious suggestions.

\section{References}

Ayadi M., Nevers T., «Un modèle Eulerien de calcul de la charge critique de flambement d'une plaque mince multicouches délaminée », Les Annales de l'ENIT, vol. 4, n 1, 1990.

Ayadi M., «Sur un algorithme pour rendre compte du contact unilatéral dans une plaque délaminée », Les Annales Maghrébines de l'Ingénieur, vol. 7, n 2, 1993.

Ayadi M., "An algorithm for computing the critical state of unilateral buckling of thin plates", European Journal of Computational Mechanics, vol. 15, $n^{\circ}$ 4, 2006, p. 341-358.

Chai H., "On the post-buckling behavior of bilaterally constrained plates", International Journal of Solids and Structures, vol. 39, n 11, 2002, p. 2911-2926.

Ciarlet P.G., Elasticité tridimensionnelle, Collection Recherches en Mathématiques Appliquées, Paris, Masson, 1986.

Ciarlet P.G., The Finite Element Method for Elliptic Problems, Series "Studies in Mathematics and its Applications", North-Holland, Amsterdam, 1978.

Ciarlet P.G., Destuynder P., «Une justification du modèle biharmonique en théorie linéaire des plaques », C. R. Acad. Sci. Paris, Sér. A 285, 1977, p. 851-854. 
600 Revue européenne de mécanique numérique. Volume $16-\mathrm{n}^{\circ}$ 5/2007

Ciarlet P.G., Introduction à l'analyse numérique matricielle et à l'optimisation, Collection Mathématiques Appliquées pour la maîtrise, Paris, Masson, 1982.

Cimetière A., «Un problème de flambement unilatéral en théorie des plaques », Journal de Mécanique, vol. 19, n 1, 1980, p. 183-202.

Cimetière A., "Méthode de Liapounov-Schmidt et branche de bifurcation pour une classe d'inéquations variationnelles », C. R. Acad. Sci. Paris, t. 300, Sér. I, n 15, 1985, p. 565-568.

Davet J., Destuynder P., « Singularités logarithmiques dans les effets de bord d'une plaque en matériaux composites ", Journal de Mécanique Théorique et Appliquée, vol. 4, 1985, p. $357-380$.

Destuynder P., Modélisation des coques minces élastiques, Collection Physique Fondamentale et Appliquée, Paris, Masson, 1990.

Do C., «Problèmes de valeurs propres pour une inéquation variationnelle sur un cône et application au flambement unilatéral d'une plaque mince ", C. R. Acad. Sci. Paris, Sér. A 280,1975 , p. 45-48.

Do C., "The buckling of a thin elastic plate subjected to unilateral conditions", Applications of Methods of Functional Analysis to Problems in Mechanics, Springer Lecture Notes, $\mathrm{n}^{\circ} 503,1976$, p. $307-316$.

Duvaut G., Lions J., Les inéquations en Physique et en Mécanique, Paris, Dunod, 1972.

Goeleven D., Montreanu D., "Asymptotic Eigenvalues and Spectral Analysis of Variational Inequalities", Communications in Applied Analysis, n 2, 1998, p. 343-372.

Goeleven D., Théra M., "Nonlinear Variational Inequalities Depending on a Parameter", Serdica Mathematical Journal, $\mathrm{n}^{\circ}$ 21, 1995, p. 1001-1017.

Kucera M., "A New Method for Obtaining Eigenvalues of Variational Inequalities: Operators with Multiple Eigenvalues", Czechoslovak Mathematical Journal, n³ 32, 1982, p. 197-207.

Quittner P., "Spectral Analysis of Variational Inequalities", Commentationes Mathematicae Universitatis Carolinae, $\mathrm{n}^{\circ}$ 27, 1986, p. 605-629.

Raviart P.A., Thomas J.M., Introduction à l'Analyse Numérique des Equations aux Dérivées Partielles, Collection Mathématiques Appliquées pour la Maîtrise, Paris, Masson, 1983.

R.C. Riddell R., "Eigenvalue Problems for Nonlinear Elliptic Variational Inequalities on a Cone", Journal of Functional Analysis, n 26, 1977, p. 333-355.

Shahwan K.W., Waas A.M., "Buckling of unilaterally constrained plates: applications to the study of delaminations in layered structures", Journal of The Franklin Institute, vol. 335, $\mathrm{n}^{\circ} 8,1995$, p. 1009-1039.

Smith S.T., Bradford M.A., Oehlers D.J., "Elastic buckling of unilaterally constrained rectangular plates in pure shear", Engineering Structures, vol. 21, n 5, 1999, p. 443-453.

Smith S.T., Bradford M.A., Oehlers D.J., "Unilateral buckling of elastically restrained rectangular mild steel plates", Computational Mechanics, vol. 26, n 4, 2000, p. 317-324. 